\title{
Metastatic Breast Cancer to Stomach Mimicking Primary
}

\section{Gastric cancer}

\begin{tabular}{|c|c|c|c|c|c|}
\hline Ava T. Ismael $^{1 *}$ & Jalal A. Jalal' ${ }^{2}$ & Sarhang Hussein ${ }^{3}$ & Nazar M.T. Jawhar ${ }^{4}$ & Muhammad Yhya Shafeek ${ }^{5}$ & Rafal Abdulrazaq $^{1}$ \\
\hline
\end{tabular}

Although breast cancer is quite common, metastases to the stomach are rare: Unlike infiltrative ductal carcinoma, invasive lobular carcinoma (ILC) has a high tendency to metastasize to the stomach. The present study reports a case of a 61-year-old female who had undergone a modified radical mastectomy of the right breast for ILC eleven years before and now presented at the clinic seeking treatment for epigastric discomfort, from sour regurgitation and belching that had persisted for three months. Gastroscopy revealed a constricting obstructing narrowed lesion in the stomach, biopsy revealed a poorly differentiated carcinoma of single cell type with areas showing Indian file arrangement; the lesion was multifocal in the stomach at which the mucosa was intact. Surgery was performed because of obstructing nature of the tumor. The diagnosis of metastatic lobular carcinoma from the breast was further established using histological and immunohistochemical markers for gross cystic disease fluid protein-15, cytokeratin (CK)7 and CK20, ER, PR.

Aim: Few cases of breast cancer metastasizing to the stomach have been reported, particularly those that have been confirmed using gastroscopy. This case highlights the importance of considering gastric metastasis of breast cancer as a differential diagnosis in patients who present with a gastric lesion and a history of breast cancer and to raise awareness of the condition.

Keywords: breast cancer; infiltrative lobular carcinoma; metastatic gastric cancer; histopathology; immunohistochemistry.

\section{Introduction}

Although breast cancer is quite common, metastases to the stomach are rare. ${ }^{1,2}$ The most frequent sites of breast cancer metastasis are the local and distant lymph nodes, brain, lung, liver and bone, with metastasis to the stomach being relatively rare. An Italian single-institution retrospective review of 980 breast cancer cases demonstrated a $0.3 \%$ incidence of gastric metastases. ${ }^{1}$ When metastasis to Stomach occurs, lobular carcinoma is by far the most common breast cancer histological type, with an estimated prevalence of $85^{3}$ to $97 \% .{ }^{4}$ However, autopsy series have reported metastatic infiltration of the uterus, peritoneum, retroperitoneum, meninges, intestine, and stomach, ${ }^{5}$ autopsies of patients with metastatic lobular cancer showed a $6-18 \%$ incidence of gastrointestinal ( $\mathrm{Gl}$ ) metastases ${ }^{6}$ suggesting that the GI lumen is a relatively common eventual site of progressive metastases. Linitisplastica of the stomach is a potential long-term sequela of metastatic breast cancer. It is essential that the clinician be aware of this pathologicentity when evaluating women, with or without a history of breast cancer, because the treatment for metastatic breast cancer presenting as linitisplastica is dramatically different from that

${ }^{1}$ Department of Clinical Analysis, College of Pharmacy, Hawler Medical University, Erbil, Iraq.

2 Department of Pathology, College of Medicine, Hawler Medical University, Erbil, Iraq.

3 Department of General Surgery, College of Medicine, Hawler Medical University, Erbil, Iraq.

${ }^{4}$ Department of Pathology, College of Medicine, Ninevah University, Ninevah , Iraq.

${ }^{5}$ Consultant in Gastroenterology and Hepatology, Ministry of Health, Iben Sena Hospital, Ninevah, Iraq.

* Correspondence: avatahir5@gmail.com 
implemented to treatprimary gastric cancer presenting as linitisplastica.

\section{Case Report}

A 61-year-old female presented to the Department of General Surgery at Rezgary Teaching Hospital of Erbil- Kurdistan region/Iraq seeking treatment for epigastric discomfort from sour regurgitation and belching that had been persisted for three months. The patient had no history of bleeding, tarry stools or associated hemorrhagic symptoms. The patient had previously undergone a modified radical mastectomy for ILC of the right breast in May 2007, with metastasis to seven axiliary lymph nodes out of twelve with no extra nodal infiltrate (T2N2MX). Her mastectomy sample immunohistochemical profile (IHC) was positive for estrogen (ERs) and progesterone receptors (PRs) and it was questionably negative for Her 2 neu (border line +2). Work up for distant metastatic disease was negative, and her complete hematology and oncology profile were unremarkable. She was treated with adjuvant chemotherapy AC (Adriamycine $60 \mathrm{mg} / \mathrm{m} 2$ and Cyclophosphomide $600 \mathrm{mg} /$ m2Q 3 Weeks' time 4, NSABP B-15 regime) with fourth cycle completed in 9/1/2007. She received Paclitaxel (Taxol $80 \mathrm{mg} / \mathrm{m} 2$ weekly for three weeks for total of four complete cycles, she completed her fourth cycle in $5 / 5 / 2007$, and then referred to adjuvant chemotherapy.

\section{Diagnosis}

Gastroscopy was done twice to the patient with interval of three weeks that revealed multiple small nodules in lesser curve of the stomach (Figure 1 A, B \& Figure 4). Multiple endoscopic biopsies were performed. Histopathological diagnosis based on hematoxylin and eosin (H\&E) staining slides revealed scattered poorly differentiated cells in the lamina propria and submucosa with focal surface ulceration, the cells were arranged in indian file pattern suggesting metastatic rather than primary diffuse gastric carcinoma of signet ring and single cell patterns (Figure 2).
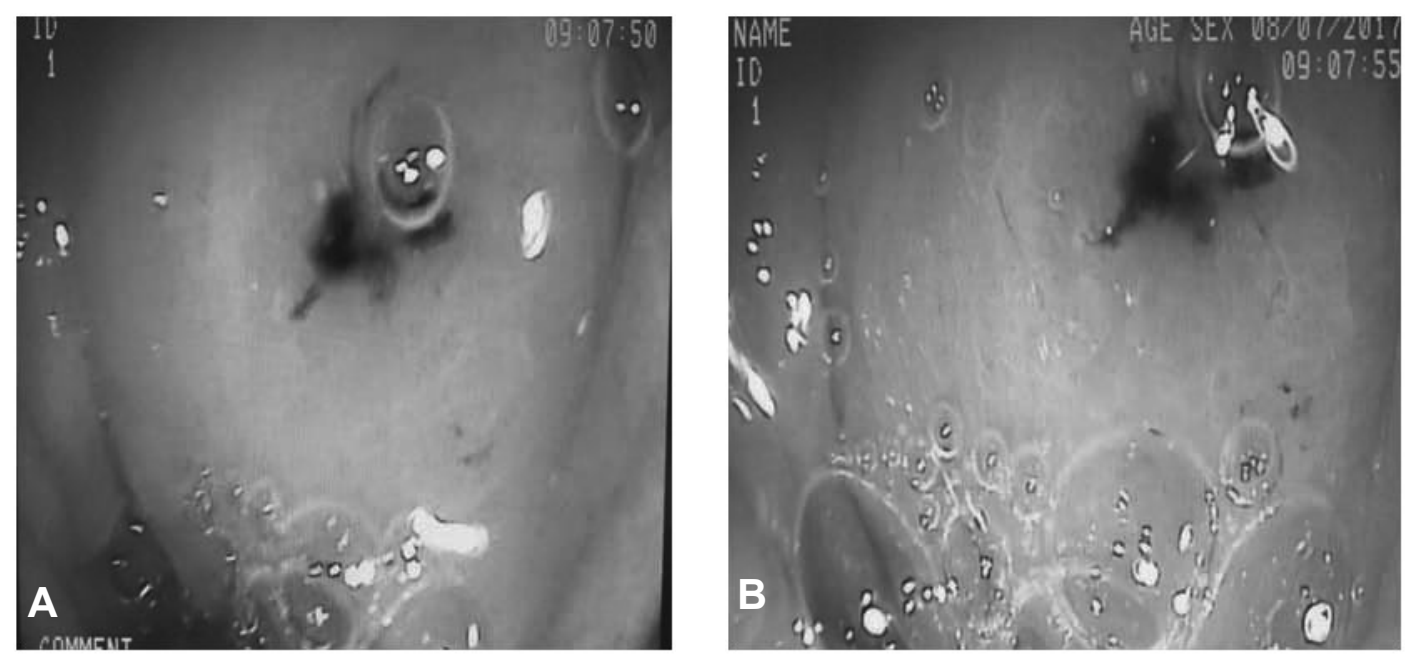

Figure 1 (A \& B): Gastroscopy revealing multiple small lesions in the body of the stomach. 

Kurdistan)

Chest, abdomen and pelvic CT with IV contrast revealed normal gastric wall thickness and no mass was detected (Figure 3). Moreover, because of limitation of tumor cells in both gastroscopic biopsies, a wide histochemical pannel was not performed apart from cytokeratin
(CK) $7^{+}$which was positive, CK20 which was negative. Based on the medical history of the patient and the findings of $\mathrm{IHC}$ and gastroscopy, suggestion of breast cancer metastasis to the stomach was made.

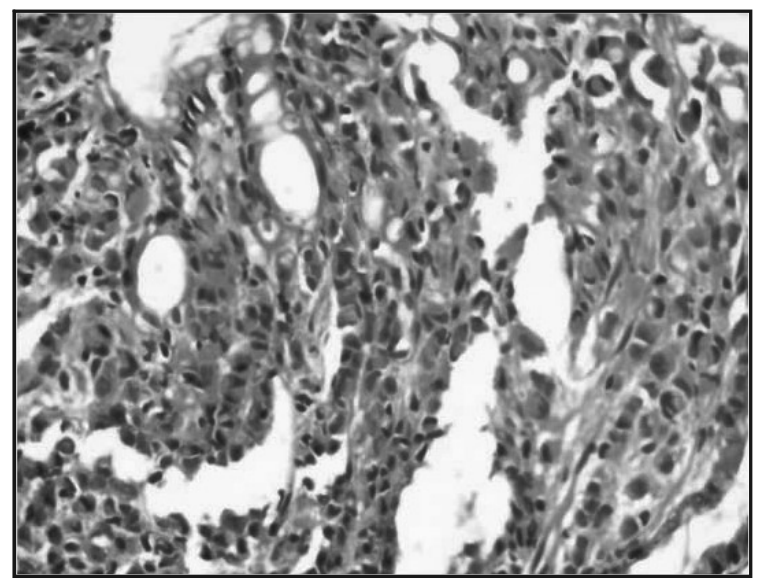

Figure 2: Histopathology revealed intact mucosa with infiltration of single cells in the lamina propria. (H\&E, X100).

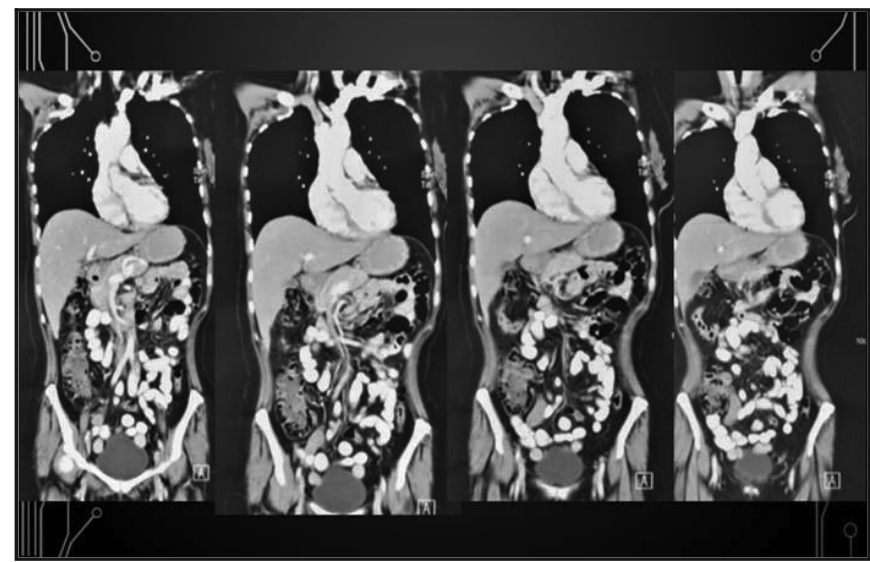

Figure 3: CT scan of abdomen revealed no mass.

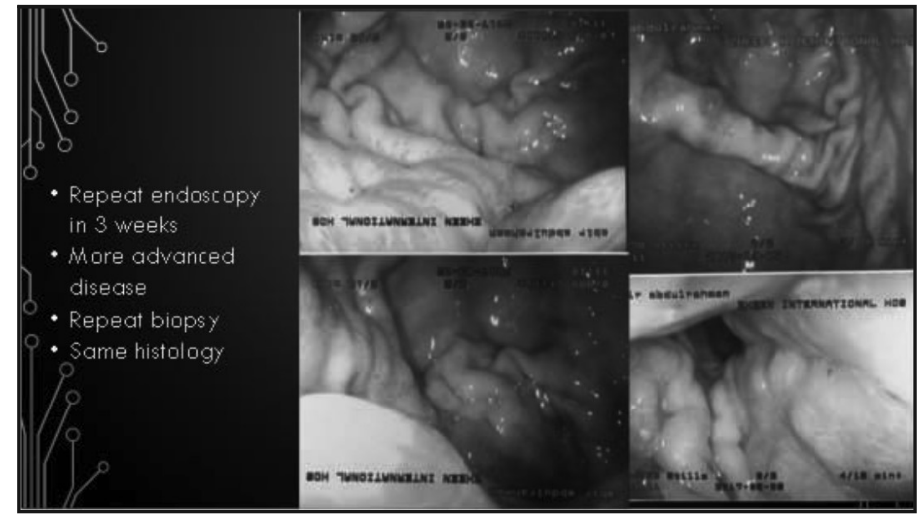

Figure 4: Repeating endoscopy and biopsy. 
The patient undergone total gastrectomy, with Roux-en-Y esophagojejunostomy reconstruction, partial colectomy (transverse colon) which was indicated due to the presence of colonic strictures with dissection of omental fat, were sequentially performed as a part of palliative surgery duo to obstruction. The patient was discharged home on postoperative day 9 after tolerating oral diet (Figure 5). Final histopathological analysis of the stomach, demonstrated diffuse infiltration with a poorly differentiated adenocarcinoma predominantly involving the muscularispropria and serosal fat (Figure 6). All five perigastric lymph nodes were tumor free. The stricture area in transverse colon showed hemorrhagic infarction and it was tumor free. The omental fat showed congestion only and it was tumor free too. Immunohistochemical stains revealed $\mathrm{CK} 7(+)$, CK20 (-), ER (+), PR (+), and E-cadherin (faintly + ), GCDFP (gross cystic disease fluid protein-15) feaures that favor a breast primary .

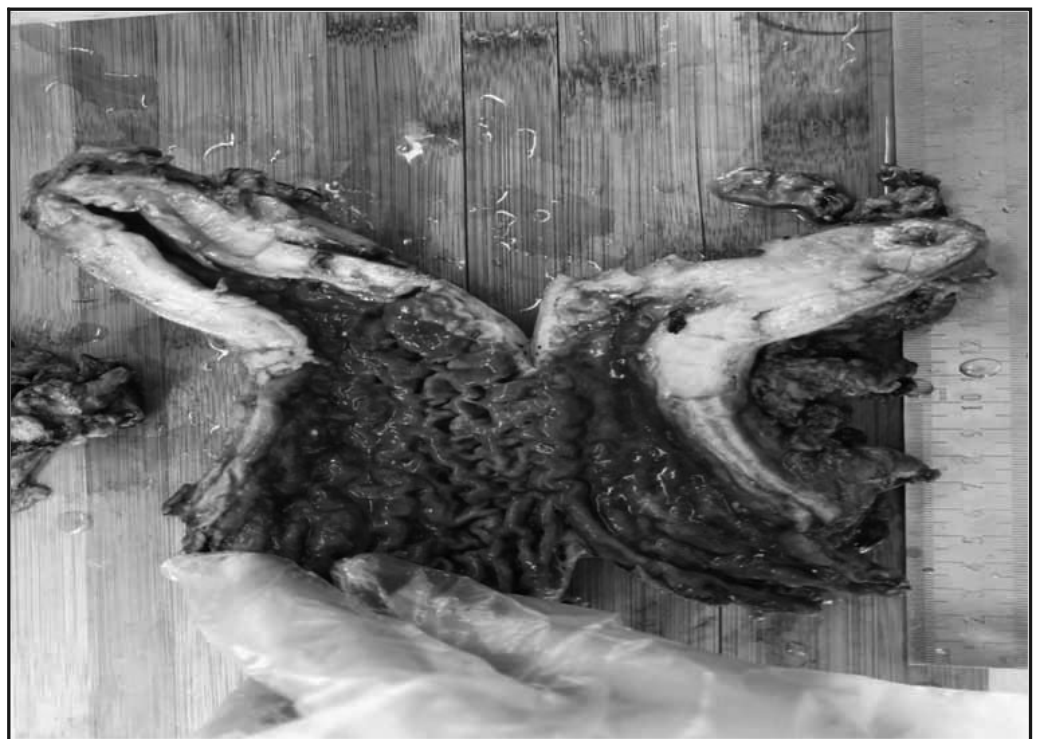

Figure 5: Gastrectomy specimen representing a constricting infiltrating mass.

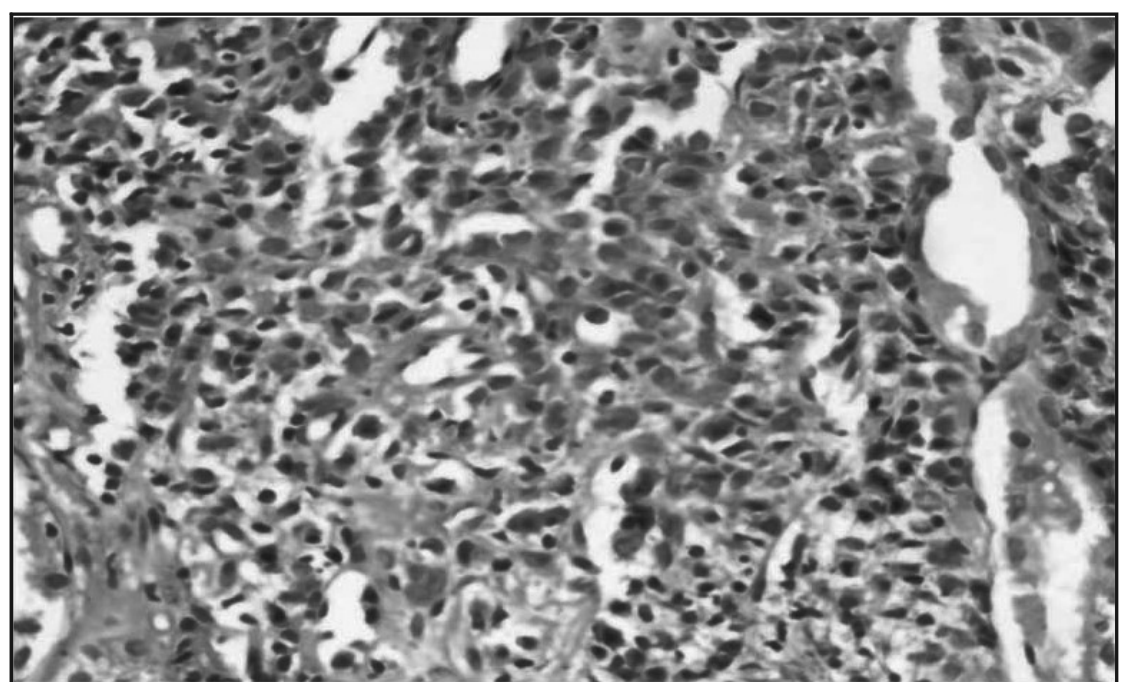

Figure 6: Final histological section of the gastrectomy specimen, showing single cells with indianfile arrangement (H\&E X100). 


\section{Discussion}

infiltrative lobular carcinoma is the second most common type of breast cancer, it accounts for $6-14 \%$ of breast cancer with distinctive biological behavior, ${ }^{8,9}$ in comparison with infiltrative duct carcinoma (IDC), ILC occurs in older age group, mostly postmenopausal with larger tumor size, low grade, ER-positive and less lympho-vascular invasion ${ }^{9,10}$ and associated with better overall and disease free survival $^{9}$ Local and distant lymph nodes, brain, lung, liver and bone are the most common sites for metastasis while metastasis to the stomach is relatively rare. ${ }^{9}$ Although studies have reported that, unlike IDC, ILC has a higher tendency to metastasize to the stomach, ovaries, meninges, pleura, skin, peritoneum, duodenum and colon. ${ }^{11,12}$ This can be explained by the presence of discohesive small cells, a phenotypic trait that characterizes ILC and loss of E-cadherin, which is observed in the majority of ILC, may lead to changes in cell-cell adhesion and advantages for growth and distant metastasis. ${ }^{13}$ Symptoms of gastric metastasis are non-specific, includes epigastric pain, hemorrhage, anorexia, dysphasia and vomiting. ${ }^{14}$ Although many methods are used to confirm the diagnosis of gastric metastases, sometimes it is difficult. The identification of gastric metastases using endoscopy is also hard. Endoscopic findings, includes observation of benign appearing lesions or ${ }^{11,15}$ diffusely infiltrative lesions. ${ }^{14}$ Histology confirms $\sim 90 \%$ of gastric metastatic lesions ${ }^{16}$ and immunohistochemical confirmation can aid for the definite diagnosis of metastases. Since ER and PR positive expression are not seen in gastric tumors so they are useful for diagnosing breast cancer metastases to the stomach ${ }^{17}$ but if the primary lesion was negative for ER and $P R$, then other markers should be performed such as CK20 and CK7 which also aids in the diagnosis. ${ }^{18}$ Also GCDFP15 isn't found in benign and malignant lesions of the stomach, thus GCDFP-15 is a specific tissue marker of breast carcinomas. ${ }^{19}$ The recommended treatment of gastric metastases from breast cancer is typically a systemic treatment. Surgical intervention should be reserved for palliation or certain cases of solitary resectable gastrointestinal tract metastases. ${ }^{20}$ The present case represents a typical metastatic breast cancer to the stomach, which was detected using gastroscopy eleven years after the surgical removal of ILC. It is essential to use GCDFP-15 and CK7/20 immunostaining for the biopsy tissue in order to identify breast cancer metastases in the stomach. When a patient has a history of ILC, endoscopic examinations should be performed carefully. Moreover, physicians should provide the clinical history of the patient to the endoscopist and the endoscopist should provide sufficient information to the pathologist in order to obtain an accurate diagnosis of breast cancer metastasis to the stomach and improve the patient's quality of life.

\section{Conclusion}

metastatic breast carcinoma to the stomach is a rare event which can be misdiagnosed as primary gastric cancer. A diagnostic approach should include clinical suspicion, repeated endoscopy, correct histpathological examination and finally immunohistochemistry is quite helpful in confirming the diagnosis.

\section{References}

1. Ambroggi M, Stroppa EM, Mordenti $P$, Biasini $C$, Zangrandi A, Michieletti E, Belloni E, Cavanna L. Metastatic breast cancer to the gastrointestinal tract: report of five cases and review of the literature. Int J Breast Cancer 2012; 439023.

2. Ciulla A, Castronovo G, Tomasello G, Maiorana AM, Russo L, Daniele E, Genova EDG. Gastric metastases originating from occult breast lobular carcinoma: diagnostic and therapeutic problems. World J Surg Oncol 2008; 6:78.

3. Hussain T, Elahi B, McManus P, Mahapatra T, and Kneeshaw $P$ J. Gastric obstruction secondary to metastatic breast cancer: a case report and literature review. J Med Case Rep 2012; 6:232. 
4. Hara F, Kiyoto S, Takabatake D, Takashima S, Aogi K, Ohsumi Sh, Teramoto $\mathrm{N}$, et al. Metastatic breast cancer to the stomach resembling early gastric cancer. Case Rep Oncol 2010; 3:142-7.

5. Harris M, Howell A, Chrissohou M, et al A comparison of the metastatic pattern of infiltrating lobular carcinoma and infiltrating duct carcinoma of the breast. Br J Cancer 1984; 50:23 -30 .

6. Ghirarduzzi A, Sivelli R, Martella E, Arrangoiz R, Papavasiliou P, Dushkin H, et al. Gastric metastasis from breast carcinoma: report of three cases, diagnostic-therapeutic critical close examination and literature review. Ann Ital Chir 2010; 81:141-6.

7. Lisa A. Whitty, David L. Crawford, Jay H. Woodland, Jitendra C. Patel, Bryce Nattier, Charles R. Thomas, Jr. Metastatic breast cancer presenting as linitisplastica of the stomach Gastric Cancer 2005; 8:193-7.

8. Arpino G, Bardou VJ, Clark GM, Elledge RM Infiltrating lobular carcinoma of the breast: tumor characteristics and clinical outcome. Breast Cancer Res 2004; 6:149-56.

9. Pestalozzi BC, Zahrieh D, Mallon E, Gusterson $\mathrm{BA}$, Price KN, Gelber RD, Holmberg SB, et al. Distinct clinical and prognostic features of infiltrating lobular carcinoma of the breast: combined results of 15 international Breast Cancer Study Group clinical trials. J Clin Oncol 2008; 26:3006-14.

10. Cristofanilli M, Gonzalez-Angulo A, Sneige N, Kau SW, Broglio K, Theriault RL, Valero V, Buzdar AU, et al. Invasive lobular carcinoma classic type: response to primary chemotherapy and survival outcomes. J Clin Oncol 2005; 23:418.

11. Almubarak MM, Laé M, Cacheux W, Arpino G, Bardou VJ, Clark GM, Elledge RM. Gastric metastasis of breast cancer: a single centre retrospective study. Dig Liver Dis 2011; 43:8237.

12. Abid A, Moffa C, Monga DK. Breast cancer metastasis to the $\mathrm{Gl}$ tract may mimic primary gastric cancer. J Clin Oncol 2013; 31:106-7.

13. Ferlicot S, Vincent-Salomon A, Médioni J, Genin $P$, Rosty C, Sigal-Zafrani B, Fréneaux $P$, et al. Wide metastatic spreading in infiltrating lobular carcinoma of the breast. Eur J Cancer 2004; 40:336-41.

14. Taal BG, Peterse H, Boot H. Clinical presentation, endoscopic features, and treatment of gastric metastases from breast carcinoma. Cancer 2000; 89:2214-21.

15. Malhotra A, Guturu P, Basim MS, Raju GS. A rare case of breast cancer metastasis presenting as linitisplastica of the stomach and colon (with videos) Gastrointest Endosc 2009; 70:552-3.

16. De Palma GD, Masone S, Rega M, Simeoli I, Donisi M, Addeo P, et al. Metastatic tumors to the stomach: clinical and endoscopic features. World J Gastroenterol 2006; 12:7326-8.

17. vanVelthuysen $M L$, Taal $B G$, van der Hoeven $\mathrm{JJ}$, Peterse JL. Expression of oestrogen receptor and loss of E-cadherin are diagnostic for gastric metastasis of breast carcinoma. Histopathology 2005; 46:153-7.

18. Tot T. Cytokeratins 20 and 7 as biomarkers: usefulness in discriminating primary from metastatic adenocarcinoma. Eur J Cancer 2002; 38:758-63.

19. Wick MR, Lillemoe TJ, Copland GT, Swanson PE, Manivel JC, Kiang DT. Gross cystic disease fluid protein-15 as a marker for breast cancer: Immunohistochemical analysis of 690 human neoplasms and comparison with alphalactalbumin. Hum Pathol 1989; 20:281-7.

20. Pectasides D, Psyrri A, Pliarchopoulou K, Floros T, Papaxoinis G, Skondra M, et al. Gastric metastases originating from breast cancer: report of 8 cases and review of the literature. Anticancer Res 2009; 29:4759-63. 